\title{
A Case Report on Fever of Unknown Origin in a School Going Child-An Uncommon Answer in a Common Question
}

\section{Dhan Bahadur Shrestha', Suju Mool ${ }^{2}$ and Sijan Karki ${ }^{1}$}

${ }^{1}$ Mangalbare Hospital, Urlabari, Morang, Nepal

2International Friendship Children’s Hospital, Maharajgunj, Kathmandu, Nepal

\section{ABSTRACT}

Fever is one of the commonest complaints in paediatrics outpatient as well as inpatient department. Fever of unknown origin (FUO) is a diagnosis of exclusion given to the febrile condition lasting more than three weeks with temperature $38.3^{\circ} \mathrm{C}$ or more with unknown cause despite of one week in patient care. Here we present a case of FUO in a child which was investigated thoroughly and managed on the line of enteric fever. However, the patient remained febrile. Hence, further investigations were undertaken and finally, bone marrow culture grew a very uncommon organism- Escherichia coli. The child was treated as per the sensitivity and responded well to treatment and was discharged after improvement. Here, we would like to present this case of FUO to highlight the possibility of bone marrow invasion by an uncommon organism as a source of fever in children.

Keywords: Fever of unknown origin (FUO), bone marrow, Escherichia coli, meropenem, enteric fever

Correspondence: Dhan Bahadur Shrestha, Mangalbare Hospital, Urlabari, Morang, Nepal. Email: medhan75@gmail.com

DOI: http://dx.doi.org/10.3126/mjsbh.v17i2.20131

Submitted on: 2018-06-04

Accepted on: 2018-06-29 


\section{INTRODUCTION}

Fever of unknown origin (FUO) is a common, perplexing and frustrating problem in all medical fields to both physician as well as patient party. This is even more relevant in paediatrics. At times, many clinical conditions can give rise to prolonged fever without apparent clinical progression of the illness. It is very important and challenging to find the cause of fever and manage accordingly. FUO was defined by Petersdorf and Beesonas in 1961 as fever lasting more than three weeks, with temperature greater than $38.3^{\circ} \mathrm{C}\left(101^{\circ} \mathrm{F}\right)$ on multiple readings and the cause of fever is unknown even after one week of hospital investigation or three outpatient visits. ${ }^{1,2}$ Recently it has been modified and defined as fever in an immunologically normal host with oral or rectal temperatures of $38.0^{\circ} \mathrm{C}\left(100.4^{\circ} \mathrm{F}\right)$ at least twice a week for more than three weeks, with no conclusive history and examination findings, and one week of inpatient investigation with no definitive cause. ${ }^{3}$ This definition is also utilised in paediatrics. Almost one third of all FUO is due to infectious causes ${ }^{1}$ but it is shifting more towards noninfectious causes. FUO, if followed and investigated with scrutiny, four usual clusters of causes can be identified viz. infections, malignancies, noninfectious inflammatory diseases (NIID) and other miscellaneous conditions. ${ }^{4}$ Many laboratory investigations in addition to routine tests are necessary to probe the possible diagnosis.

Infectious causes form the bulk of cause of FUO and further analysis generally show uncommon bacterial or viral illnesses as the cause. In this regard, here we found a case of FUO associated with bone marrow invasion by $E$ coli. Interestingly, there was no growth of $E$. coli neither in blood, nor in urine, despite repeated blood as well as urine cultures. There were no associated clinical symptoms except for fever.

\section{CASE REPORT}

Seven years' boy from Kailali, Nepal, a second product of non-consanguineous marriage, developmentally normal child, with no significant past history, had presented to our hospital with the complaint of fever for 10 days. The fever was intermittent with maximum recorded temperature of $1040 \mathrm{~F}\left(40^{\circ} \mathrm{C}\right)$. There was associated chills but no rigors. He had mild headache, cough as well as some general malaise. He had no other complaints. $\mathrm{He}$ was treated previously with oral antibiotics from a local clinic with no improvement. At the time of presentation, he was conscious and febrile. Rest of the vital parameters were within normal limits. His anthropometry was normal for his age and sex. There were no significant findings on rest of the general examination. On per abdominal examination, there was hepatomegaly, with liver being palpable $3 \mathrm{~cm}$ below the costal margin in mid clavicular line which was non-tender, firm in consistency with regular borders and margins. However, spleen was not palpable and rest of the systemic examinations were all normal.

In our scenario, enteric fever is an extremely common cause of fever, perhaps because of poor hygiene and sub optimal water supply. Hence, the child was initiated with investigations in the line of enteric fever. Treatment was started with broad spectrum antibiotic Ceftriaxone in view of high prevalence of Nalidixc Acid Resistant Salmonella Typhi. His laboratory report showed haemoglobin $10.9 \mathrm{gm} \%$, leukocyte count of $8500 / \mathrm{mm}^{3}$ with neutrophils (24\%), lymphocytes (74\%), eosinophils ( $2 \%$ ), and platelets $228000 / \mathrm{mm}^{3}$ The peripheral blood smear was unremarkable. Widal test came negative and the blood culture was sterile. Thus, with no laboratory evidence of enteric fever and child not showing improvement to treatment, enteric fever was presumably ruled out. The child 
remained febrile; however, he did not develop any other features.

As the child remained febrile, further investigations were undertaken to look for evidence of other common infectious conditions in our geographical region. However, Leptospira antibody, Brucella antibody, rK-39, Mantoux test were all negative. Chest $\mathrm{x}$ ray was also normal. Further evaluation was done with ultrasound abdomen to look for any occult collection of fluid or pus which we could not get. Further investigation was done with CECT abdomen to try to localise the cause of infection. However, CECT abdomen was also unremarkable. The child continued to remain febrile over the period of next two weeks. In view of FUO, we planned for bone marrow aspiration and culture to rule out any haematological malignancy or any other occult infections. Bone marrow aspiration study showed myeloid to erythroid ratio of 2:1 with erythroid series showing normoblastic maturation, myeloid series showing normal maturation with eosinophilic predominance (42\%). Megakaryocytic series was normal in morphology and distribution with no increase in blast cells.

But surprisingly, bone marrow culture grew E. coli. Isolated E. coli was sensitive to meropenem, gentamicin and amikacin and resistant to rest of the common antibiotics tested including cephalosporins. This was a rare case where bone marrow culture growth occurred with E. coli. As per the sensitivity pattern shown, we started this child on intravenous meropenem. Remarkably, after 48 hours of the antibiotic, he became afebrile. His headache was also relieved and he became completely asymptomatic. In view of the rarity of the growth of E. coli in bone marrow, we did extensive literature search. However, we could not find any publications mentioning growth of E. coli in bone marrow. As there was no guideline for the duration of antibiotics in such case, we continued with intravenous meropenem for 14 days, despite the child being asymptomatic on day 2 . After the completion of antibiotics course, the child was discharged and he is presently well and under regular follow up.

\section{DISCUSSION}

In children, especially the younger age groups, UTI is quite a common cause of fever and generally, $E$. coli is the organism grown. ${ }^{4-6} E$. coli is sometimes grown in blood culture of children with sepsis. However, there has been no report of E. coli grown in bone marrow culture. Bone marrow culture is very important for looking for occult infections as well as haematological malignancies. Bone marrow culture positivity for salmonella is extremely high when blood culture remains sterile for salmonella. Hence, in cases of FUO, bone marrow aspiration study and culture is crucial, especially when there suspicion of either infections or haematological malignancies.

First notable diagnostic definition of FUO was given by R. B. Petersdorf and Beesonas in 1961 and has been followed for decades. ${ }^{1}$ Though due to easy and wide availability of laboratory facilities, common causes of fever can be easily ruled out and fever without a source can be labelled as fever of unknown origin by at least eight days history of fever. ${ }^{3}$ Recently FUO has been defined as fever of $38.0^{\circ} \mathrm{C}\left(100.4^{\circ} \mathrm{F}\right)$ at least twice a week for more than three weeks, with no conclusive history and examination findings, and one week of inpatient investigation with no definitive cause. ${ }^{3}$ Only in about one third of total cases of FUO, cause could be pointed out and managed accordingly. 5 But due to recent advances in diagnostic modalities, in about $90 \%$ of cases cause can be identified. ${ }^{3}$ Many studies from developing countries show infection (36\% to $78 \%$ ) as the commonest cause of FUO, followed by other causes such as malignancy $(2 \%$ 
to $12 \%)$, collagen-vascular diseases ( $2 \%$ to $21 \%)$, noninfectious causes ( $2 \%$ to $50 \%$ ), and unknown $(12 \% \text { to } 29 \%)^{3,6}$ In a multi-centric prospective study, primary bacteraemia as a cause of febrile illness was found in only $3.2 \%$ of total cases, commonly due to E. coli, Streptococci, Salmonella. In majority $(61.3 \%)$ of acute undifferentiated febrile illness cases, cause was unknown and among the identifiable causes rickettsial infection, influenza and dengue were common ones. ${ }^{5}$ Another prospective study showed infection as the commonest cause of admission in hospital as acute febrile illness and E. coli positive in urine accounted significant number of cases. ${ }^{7}$

Careful detailed systemic and logical history and thorough examination with appropriate laboratory investigations and its proper interpretation is vital to reach the definitive cause for fever. Patient or care giver should be enquired about onset of fever and its documentation using thermometer is a must. Special emphasis should be given to pattern, its periodicity and associated symptoms and relation with antipyretics administration, which may bear diagnostic significance. In addition, age, race, ethnicity, family history and address give clue to significant causes of FUO. For example, like periodic fever runs in family and some fevers are endemic to some place, likewise the travel and contact history are also relevant.3,8 FUO is the uncommon presentation of common diseases so first common conditions should be ruled out before jumping to rarity. Also, pseudo FUO should be differentiated from FUO. 8,9

Battery of laboratory tests may be needed for a case of fever. The rationale for investigations should be defined and performed in logical sequence, ordering common tests first. A simple complete blood cell count $(\mathrm{CBC})$ and differential count with peripheral smear is vital in fever and may suggest an infectious or a haematological malignancy. 3,8
Blood culture should always be performed before initiation of antibiotics. Urine routine and culture are other important tests. UTI is a common cause for FUO in children and should always be looked for. 5,6 Respiratory infections are also common cause of FUO and hence chest x-ray is required to rule out infections like tuberculosis, especially in our parts of world. Similarly, Widal test and stool routine and culture are required to rule out enteric fever or gastroenteritis as the cause of fever. If there are features of neurological derangements, then CSF analysis may be of value in ruling out meningoencephalitis. Sometimes, abdominal ultrasound is required to rule out pocket of pus collection or mesenteric lymphadenopathy as a cause of fever. Similarly, other tests like antinuclear antibodies rule out connective tissue diseases. Special consideration must be given to connective tissue disorders, especially in an adolescent girl. Sometimes, rare infections may also be required to be looked for after ruling out common infections. The role of bone marrow aspiration is of immense importance to rule out occult infections as well as haematological malignancies. Though in every case cause may not be identifiable, every attempt must be done to look for it.

Here in this case, the boy presented with history of unrelenting fever which did not respond to appropriate treatment. After doing all laboratory work up diagnosis was uncertain and fever was persisting and hence bone marrow aspiration was done which later showed the growth of E. coli which was sensitive to meropenem. With meropenem and supportive management, the child became afebrile.

\section{CONCLUSIONS}

In any case of FUO, patient should be evaluated thoroughly with appropriate history and logical examination. Appropriate investigations should be 
undertaken one after another, based on suspicion to localise the cause and manage accordingly. Generally, if we follow the logical stepwise approach, even if we do not reach the diagnosis, we can at least rule out many relevant pathological conditions which may have caused fever. And sometimes, even rare conditions may present as a cause of common symptom of fever. Hence, investigations for FUO should always follow logically in sequence to arrive at early diagnosis so that appropriate management may be done.

\section{ACKNOWLEDGEMENT}

We would like to acknowledge the consultants and all the staffs of Paediatric department of Shree Birendra Hospital for their direct or indirect help during management of patient and case preparation.

To cite this article: Shrestha DB, Mool S, Karki S. A case report on fever of unknown origin in a school going child - an uncommon answer in a common question. MJSBH. 2018;17(2):58-63.

Conflict of Interest: None declared

\section{REFERENCES}

1. Petersdorf RB, Beeson PB. Fever of unexplained origin: report on 100 cases. Medicine. 1961;40:1-30. DOI: https://doi.org/10.1097/00005792-196102000-00001

2. Knockaert DC, Vanderschueren S, Blockmans D. Fever of unknown origin in adults: 40 years on. J Intern Med. 2003;253:263-75.

DOI: https://doi.org/10.1046/j.1365-2796.2003.01120.x PMid:12603493

3. Antoon JW, Potisek NM, Lohr JA. Pediatric fever of unknown origin. Pediatrics in Review. 2015;36:380. DOI: https://doi.org/10.1542/pir.36-9-380 PMid:26330472

4. Jacobs RF, Schutze GE. Bartonella henselae as a cause of prolonged fever and fever of unknown origin in children. Clin Infect Dis. 1998;26:80-4.

DOI: https://doi.org/10.1086/516256

5. Leelarasamee A, Chupaprawan C, Chenchittikul M, Udompanthurat S. Etiologies of acute undifferentiated febrile illness in Thailand. J Med Assoc Thai. 2004;87(5):464-72.

PMid:15222513

6. Kejariwal D, Sarkar N, Chakraborti SK, Agarwal V, Roy S. Pyrexia of unknown origin: a prospective study of 100 cases. J Postgrad Med. 2001;47:104.

PMid:11832599

7. Abrahamsen SK, Haugen CN, Rupali P, Mathai D, Langeland N, Eide GE, et al. Fever in the tropics: aetiology and case-fatality-a prospective observational study in a tertiary care hospital in South India. BMC infectious diseases. $2013 \mathrm{Jul} ; 13(1): 355$.

DOI:https://doi.org/10.1186/1471-2334-13-355

PMid:23899336 PMCid:PMC3750507

8. Hague R. Fever of unknown origin- investigation and management. Current Paediatrics. 2001;11:445-51. DOI: https://doi.org/10.1054/cupe.2001.0226 
9. Tolan Jr RW. Fever of unknown origin: a diagnostic approach to this vexing problem. Clinical pediatrics. 2010 Mar;49(3):207-13.

DOI: https://doi.org/10.1177/0009922809347799 